\title{
Atomically Precise Silver Clusters as New SERS Substrates
}

\author{
Indranath Chakraborty, ${ }^{\dagger}$ Soumabha Bag, ${ }^{\dagger}$ Uzi Landman, ${ }^{\dagger}$ and Thalappil Pradeep ${ }^{*}{ }^{\dagger}$ \\ ${ }^{\dagger}$ DST Unit of Nanoscience (DST UNS) and Thematic Unit of Excellence (TUE), Department of Chemistry, Indian Institute of \\ Technology Madras, Chennai 600 036, India \\ ${ }^{*}$ School of Physics, Georgia Institute of Technology, Atlanta, Georgia 30332, United States
}

\section{Supporting Information}

ABSTRACT: An atomically precise silver cluster, $\mathrm{Ag}_{152}$ protected with thiolate ligands, was used as a surface-enhanced Raman scattering (SERS) substrate. The cluster shows intense enhancement of Raman signals of crystal violet with an enhancement factor of $1.58 \times 10^{9}$. Adaptability of the substrate for a wide range of systems starting from dyes to biomolecules is demonstrated. Solid-state drop casting method was used here, and SERS signals were localized on the $\mathrm{Ag}_{152}$ crystallites, confirmed from Raman images. Excellent periodicity of clusters, their plasmonic nature, and absence of visible luminescence are the main reasons for this kind of large

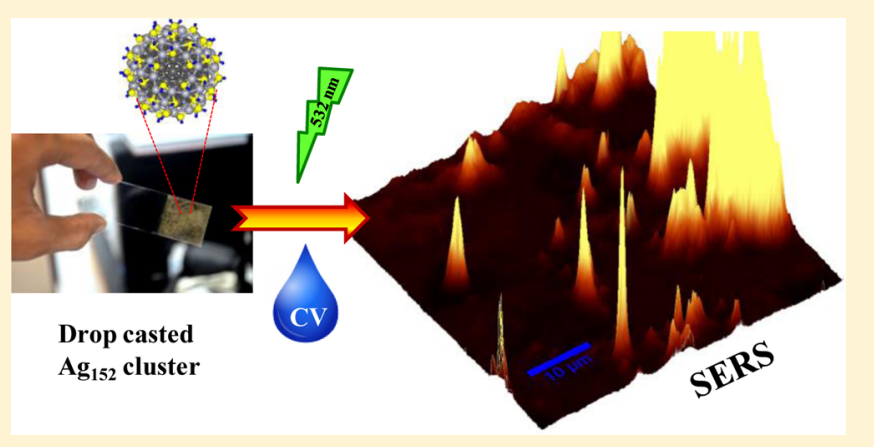
enhancement. SERS was compared with smaller clusters and larger nanoparticles, and the size regime of $\mathrm{Ag}_{152}$ was found to be optimum. Several control experiments were done to understand the SERS activity in detail. The method has wide adaptability as the cluster can be easily drop-casted on any surface like paper, cotton, and so forth to produce effective SERS media. The work suggests that atomically precise clusters, in general, can show SERS activity.

SECTION: Physical Processes in Nanomaterials and Nanostructures

$\mathrm{N}$ anoscale atomic clusters of noble metals, especially gold and silver, are emerging materials with novel properties. ${ }^{1}$ While much of the research effort in this area is focused on gold

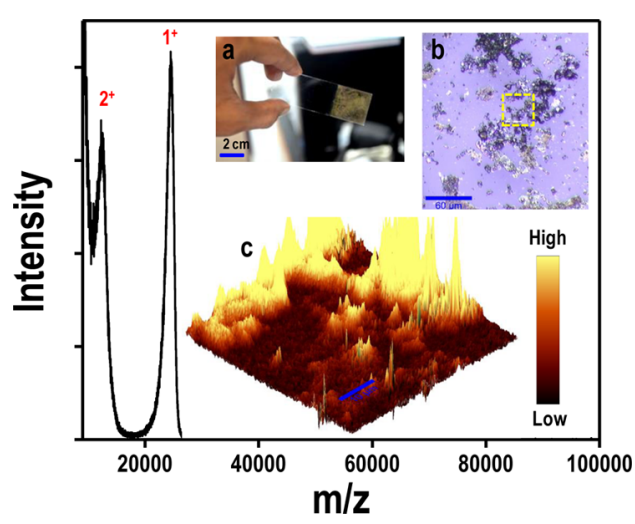

Figure 1. MALDI mass spectrum of a purified $\mathrm{Ag}_{152}$ cluster sample in toluene. A DCTB matrix was used. It gives a sharp molecular ion peak at $m / z 24610 \pm 50$ along with a prominent dication peak at $m / z 12$ $300 \pm 30$. Inset (a) shows a photograph of a drop-casted film of a $\mathrm{Ag}_{152}$ cluster on a glass slide. Multiple drop-castings can be done to increase film thickness. Inset (b) shows the optical image of a dropcasted film with crystal violet $(\mathrm{CV})$ as an analyte. The image shows microcrystallites of the cluster. A selected area of (b) was chosen for Raman imaging, and the corresponding 3D view (with Raman intensities in the narrow window) is given in inset (c). aggregates, ${ }^{2-8}$ studies of silver clusters are relatively scarce, though several well-characterized systems have been explored rather recently. ${ }^{9-13}$ Characteristic features in optical absorption and visible-to-near-infrared luminescence have made these molecular systems new probes for analytical methodologies using spectroscopy. As the nuclearity (number of atoms in the cluster) increases, light emission shifts to the red and nearinfrared regions, and the absorption spectrum resembles that of nanoparticles (NPs), with characteristic plasmon absorptionlike features. The emergence of plasmonic properties in atomically precise clusters has been demonstrated with $\mathrm{Ag}_{152}{ }^{9}$. This size regime is one at which visible luminescence nearly disappears, and its absence may be advantageous for scattering-based spectroscopies. In the past several years, atomically precise clusters have been used for metal ion sensing, ${ }^{14}$ biolabeling, ${ }^{3,15}$ cancer targeting, ${ }^{16}$ catalysis, ${ }^{17}$ and many other applications. ${ }^{18}$ The small size, reduced or absent cytotoxicity, diverse functionalization, and incorporation ability in various matrixes are among the specific advantages of such systems. The addition of other properties to the above list, in particular, surface-enhanced Raman scattering (SERS), ${ }^{19-27}$ promises to increase efforts aimed at understanding and utilizing these new materials.

Received: July 8, 2013

Accepted: July 30, 2013

Published: July 30, 2013 


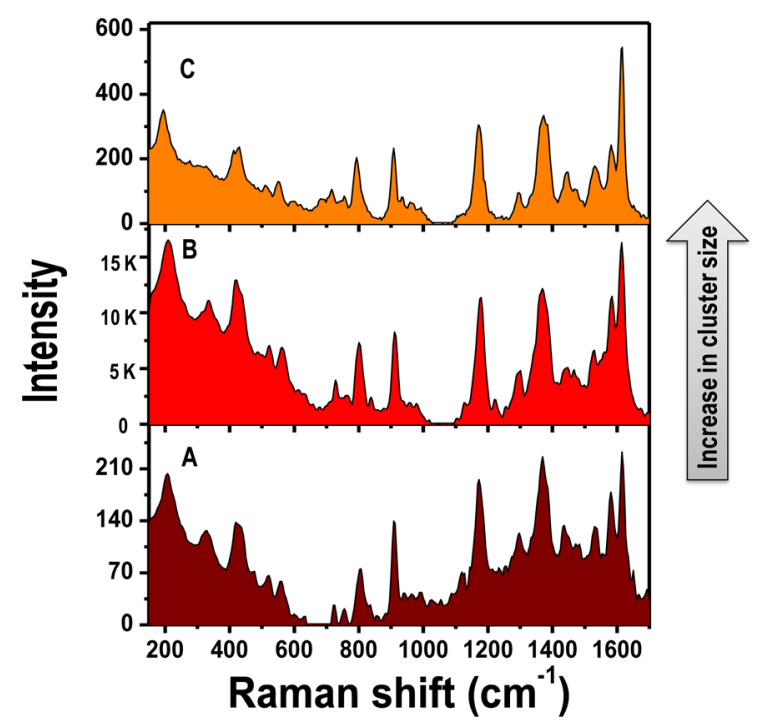

Figure 2. Comparative SERS spectra of $\mathrm{CV}$ on a drop-casted film of a $\mathrm{Ag}_{55}$ cluster (A), $\mathrm{Ag}_{152}$ cluster (B), and 3-4 nm Ag NP (C). Similar experimental conditions and the same laser intensities were used for all of the cases shown. A PET-protected $\mathrm{Ag}_{55}$ cluster and NPs were used here. The $50 \mu \mathrm{M} \mathrm{CV}$ was taken as the analyte. The spectra show unprecedented enhancement of the $\mathrm{Ag}_{152}$ cluster compared to the $\mathrm{Ag}_{55}$ and the silver NP (compare the $y$ axes).

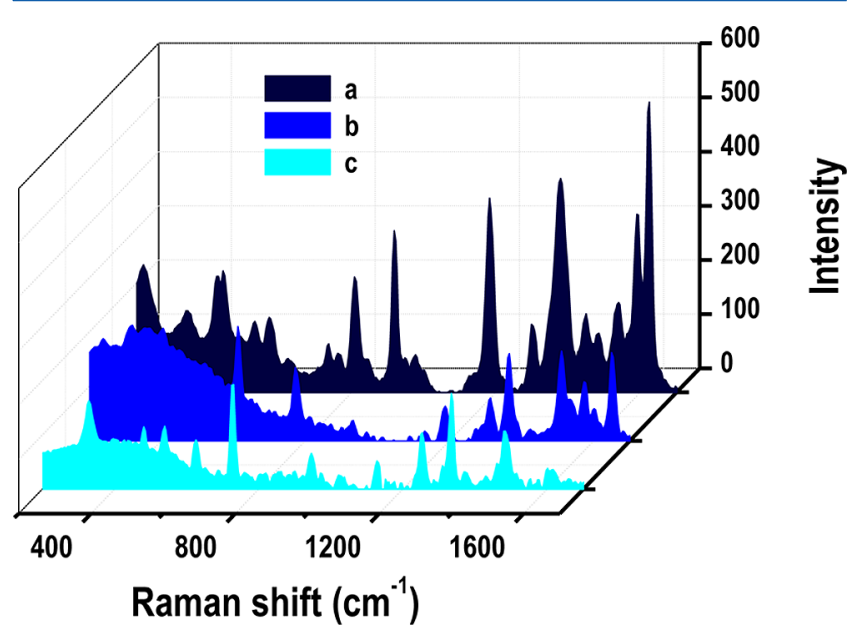

Figure 3. The adaptability with other analytes is shown here; $a, b$, and c show the SERS spectra of CV, rhodamine 6G (R6G), and adenine, respectively. Characteristic Raman features of each of the analytes are present. The $10^{-7} \mathrm{M} \mathrm{CV}$ and R6G and $10^{-6} \mathrm{M}$ adenine were used for the experiments. Instrumental parameters were kept constant.

In this Letter, we show the occurrence of intense SERS in a $\mathrm{Ag}_{152}$ cluster. This is the first report of the observation of SERS in monolayer protected silver clusters, and therefore, it extends the scope of applications of atomically precise clusters. The results presented here using several analytes confirm the adaptability of the substrate for diverse systems. Besides solidstate measurements, we demonstrated the use of this material for solution-phase studies as well. The enhancement in the solid state is attributed to the creation of hot spots at specific regions spread over the crystallites. The observation of an enhancement factor (EF) on the order of $1.5 \times 10^{9}$ for $\mathrm{Ag}_{152}$ suggests a significant cost savings associated with the use of these materials in comparison to typical silver NP systems composed of $\sim 10000$ atoms. The results of our study can be understood on the basis of reports where molecular systems have been predicted to exhibit pronounced Raman enhancement. $^{28}$

As the characterization and properties of the $\mathrm{Ag}_{152}$ cluster have been reported previously, ${ }^{9}$ we present here only the most essential features that are of relevance to this study. The cluster shows a well-defined MALDI mass spectrum (Figure 1A) at $\mathrm{m} /$ $z 24610$ with a prominent dication feature at $\mathrm{m} / z 12300$ with trans-2-[3-(4-tert-butylphenyl)-2-methyl-2-propenylidene] malononitrile (DCTB) as the matrix. ${ }^{10,29}$ The cluster exhibits faceted crystallites in SEM images (Figure S1, Supporting Information), which also show the expected elements and the intensities (data not shown) in energy-dispersive analysis of Xrays (EDAX). Transmission electron microscopic (TEM) images confirm the high uniformity of the cluster size and shape (Figure S1, Supporting Information) and also suggest excellent periodicity, which might be a reason for generating hot spots on crystallite surfaces for the observed SERS activity.

An optical image of a portion of a drop-casted film is displayed in Figure $1 b$, showing the microcrystalline nature of the cluster. The Raman spectrum of crystal violet $(\mathrm{CV})$ on a drop-casted film of $\mathrm{Ag}_{152}$ is shown in Figure S2a (Supporting Information). The spectrum shows all of the features of bulk $\mathrm{CV}$, and comparison of both is given in Figure S2 (Supporting Information). The observed enhancement factor ${ }^{30,31}$ ( $\mathrm{EF}=$ $1.58 \times 10^{9}$, details are in Supporting Information Table 1) is unprecedented, and it is almost 3-4 orders of magnitude greater than the corresponding silver NP system reported in the literature. ${ }^{32}$ The SERS is localized on the crystallites of $\mathrm{Ag}_{152}$, as confirmed from the Raman image shown in Figure 1c. The Raman image was collected based on the intensities in the 1605-1646 $\mathrm{cm}^{-1}$ window. An image collected for a wider window $\left(150-1700 \mathrm{~cm}^{-1}\right.$, in which $\mathrm{CV}$ has its characteristic signals) also shows (Figure S3, Supporting Information) a similar pattern. The direct correlation between the Raman image and the optical image confirms (Figure S4A and B, Supporting Information) the existence of active sites on the crystallites. The corresponding spectra from dark green and light yellow regions (Figure S4C and D, Supporting Information) reflect the presence and absence of $\mathrm{CV}$ characteristics, respectively, which proves that SERS sites are the microcrystals of $\mathrm{Ag}_{152}$.

The SERS EF of the $\mathrm{Ag}_{152}$ clusters is compared in Figure 2 with that of the corresponding silver NP system with PET (phenylethanethiol, in the thiolate form) protection. A film of PET-protected plasmonic NPs of 3-4 nm diameter, prepared through the use of a similar procedure to that used for the $\mathrm{Ag}_{152}$ cluster, exhibits an EF of $7.5 \times 10^{5}$. Additionally, we find that a similarly prepared $\mathrm{Ag}_{55}$ cluster system, also protected with PET, shows a reduced EF of $2.7 \times 10^{5}$. The unusually large SERS enhancement of the $\mathrm{Ag}_{152}$ clusters compared to larger NPs and smaller clusters may originate from the periodic arrangement of the nanocrystallites, which brings about the formation of hot spots between the clusters. The absence of emission in the visible region assists the acquisition of the Raman spectrum, which may be an issue for smaller and inherently luminescent clusters. $^{10,12,33}$ Another source for the high SERS enhancement of the $\mathrm{Ag}_{152}$ clusters is their optical absorption spectrum, which is comparable to the plasmon resonance of the silver NPs, and it overlaps with the excitation line. From the foregoing, we would like to emphasize that the observed EFs are not of the isolated clusters but of their solid-state analogues, which correspond to aggregated structures. However, the nature of 

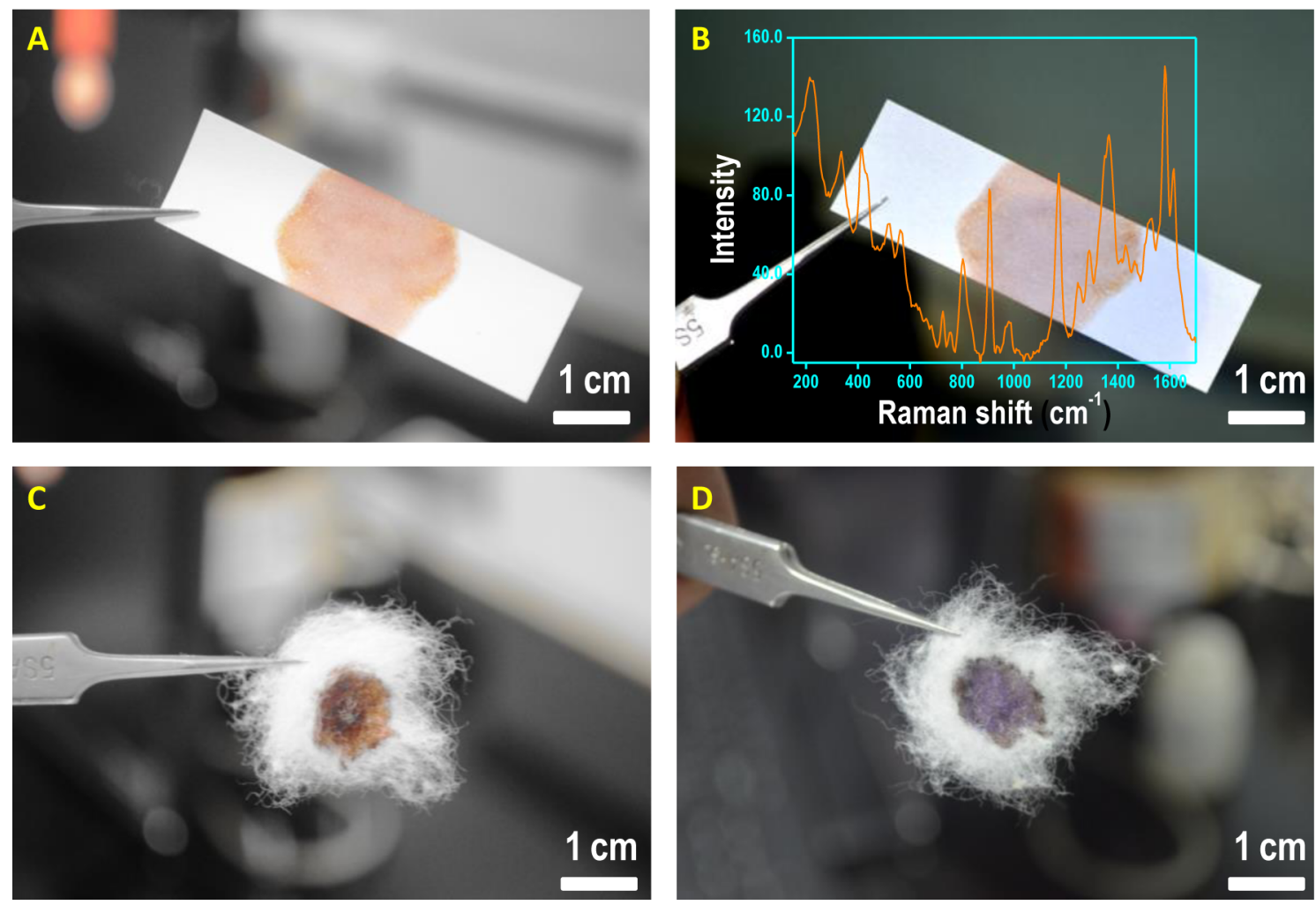

Figure 4. Photographs of $\mathrm{Ag}_{152}$ clusters coated on paper $(\mathrm{A}, \mathrm{B})$ and cotton $(\mathrm{C}, \mathrm{D})$ before $(\mathrm{A}, \mathrm{C})$ and after $(\mathrm{B}, \mathrm{D}) \mathrm{CV}$ was drop-casted. The inset of $(\mathrm{B})$ shows the Raman spectrum of $\mathrm{CV}$ taken from the cluster-coated paper substrate.

the intercluster regions responsible for enhancement cannot be evaluated from the current experiments as they are under subdiffraction limits and are not probed here.

Several control experiments were done to understand how the nature of the films affects the SERS property of the clusters. A decrease in the concentration of the $\mathrm{Ag}_{152}$ clusters by dilution of the solution used for drop-casting (Figure S5, Supporting Information) or an increase in the concentration by performing multiple coatings (Figure S6, Supporting Information) decreases the SERS intensity. Variations in the coverage or concentration suggests that a specific morphology and number of particles are important, which is in accordance with previous observations. ${ }^{28}$ Besides the solid-state drop-casting method, we have also studied SERS in the solution phase (data not shown), where the $\operatorname{EF}\left(9.5 \times 10^{8}\right)$ is somewhat lower. The concentration dependence of the analyte (Figure S7, Supporting Information) shows the lower detection limit of $\mathrm{CV}$ to be $10^{-9} \mathrm{M}$. The corresponding spectrum has been expanded 10 times to allow clear inspection of the features. The intensity of the $1379 \mathrm{~cm}^{-1}$ peak is plotted against the concentration, which shows the limit of detection. As clusters are susceptible to electron- and laser-induced damage, we characterized the film before and after SERS measurements. Although UV/vis does not show any (Figure S8, Supporting Information) significant change, we could see visible damage of the film morphology after each experiment (data not shown). Along with PET-protected silver NPs, the SERS of $\mathrm{Ag}_{152}$ clusters was compared also with that of citrate-capped NPs. In the latter case, an expected enhancement $\left(\mathrm{EF}=1.98 \times 10^{6}\right)$, as reported in the literature, ${ }^{32}$ was seen. The TEM image and corresponding UV/vis spectra for the citrate- and PET-capped NPs are given in Figure S9 (Supporting Information).
Another advantage of cluster-based materials is that they are soluble in diverse media, and as a result, effective substrates can be prepared easily. The cluster can be coated on paper (Figure $4 \mathrm{~A})$, cotton (Figure 4C), silk, as well as other materials, and such active substrates can be dipped in analyte solutions, and SERS measurements can be made. The clusters get coated uniformly over the substrates, and the amount of silver loaded to get complete coverage is much smaller in comparison to that for plasmonic NPs, which contributes to the reduced cost of such subtrates. Luminescence from paper and cotton, which contain cellulose and other organic matter, can pose difficulties for SERS detection. Consequently, glass was chosen as a better substrate. It is important to note that clusters can be used to create patterns, as described in our previous study, on gold clusters, $^{34}$ and such patterned surfaces will be useful for diagnostics.

To check whether the SERS is restricted to $\mathrm{CV}$, other analytes were tried. Rhodamine 6G (R6G), which is another often-used analyte for SERS experiments, shows a SERS signal even at $10^{-7} \mathrm{M}$ concentration (Figure $3 \mathrm{~b}$ ). Similar enhancement was found for the biomolecule, adenine (Figure 3c). The corresponding EFs are $1.08 \times 10^{8}$ and $1.30 \times 10^{8}$ for R6G and adenine, respectively. The most intense bands in these cases and their intensities are given in Table 2 (Supporting Information). The Raman spectra consist of all of the characteristic features of R6G and adenine, as reported in the literature. $^{35,36}$ These results suggests that the $\mathrm{Ag}_{152}$ cluster system can be employed as a universal SERS substrate.

It may be noted that the resonance Raman (RR) ${ }^{37-39}$ effect, strongly sensitive to the excitation energy, ${ }^{40}$ cannot be avoided for the case of $\mathrm{CV}$ as the excitation wavelength is $532 \mathrm{~nm}$. Even for R6G, it can interfere, ${ }^{39}$ but adenine, which does not show a $\mathrm{RR}$ effect ${ }^{41-44}$ for this excitation, also shows similar enhance- 
ment, suggesting that the enhancement here is principally due to SERS. To further prove the point, additional measurements were carried out at $633 \mathrm{~nm}$ excitation. For R6G, CV, and adenine (all measured at $5 \mu \mathrm{M}$ concentration, drop-casted), a glass substrate gave spectra comparable to those reported here with EFs of $1.1 \times 10^{8}, 1.6 \times 10^{9}$, and $1.4 \times 10^{8}$, respectively. Comparative spectra due to 532 and $633 \mathrm{~nm}$ excitations are given in Figure S10A (Supporting Information). Hence, the enhancement here is largely due to SERS.

In summary, the results presented in this Letter show that atomically precise clusters are new candidates for SERS measurements. Their plasmon-like optical feature, crystalline nature (of the individual nanoclusters and their assembly), and the absence of visible luminescence are among the main reasons for this enhancement. Unprecedented EFs, broad applicability to a number of analytes, and adaptability to various substrates, including glass, paper, and cotton, suggest the possibility for surface functionalization, which makes this system highly useful along with the large reduction in cost in comparison to plasmonic nanosystems.

\section{EXPERIMENTAL METHODS}

Details of the chemicals used are given in the Supporting Information. $\operatorname{Ag}_{152}(\mathrm{PET})_{60}$ [PET: phenylethanethiol, in the thiolate form] was synthesized by a solid-state method. ${ }^{11}$ Briefly, the method involves grinding of $\mathrm{AgNO}_{3}$ with PET in a molar ratio of 1:5.3 to form silver thiolate. Subsequent addition of $0.675 \mathrm{mmol}$ of $\mathrm{NaBH}_{4}$ in the solid state and continuous grinding created the cluster, which was initially extracted in ethanol to remove the unreacted thiol by centrifugation followed by re-extraction of the residue with toluene (additional details are given in the Supporting Information). $\mathrm{Ag}_{55}$ clusters and Ag NPs protected with PET were also prepared. The clusters were characterized by a number of analytical methods (details are in the Supporting Information).

This cluster solution was drop-casted on glass coverslips to create SERS-active substrates. Analyte solutions were dropcasted on them and were left to dry in ambient laboratory conditions. Raman investigations were done with 532 and 633 nm excitation using a WITec confocal Raman microscope (details are given in the Supporting Information). All of the spectra were collected after background correction to exclude fluorescence interference. ${ }^{40}$ For comparison, data without background correction is given in Figure S10B (Supporting Information) (for $532 \mathrm{~nm}$ excitation).

\section{ASSOCIATED CONTENT}

\section{S Supporting Information}

Details of experimental procedures, EF calculation and characterization of $\mathrm{Ag}_{152}$ clusters and other materials, one-toone correlation of the Raman image with the optical image, control experiments with variation in concentrations and coatings, comparison with data from nanoparticles, images from different substrates, SERS spectra at two excitations, and data without background correction. This material is available free of charge via the Internet at http://pubs.acs.org.

\section{AUTHOR INFORMATION}

\section{Corresponding Author}

*E-mail: pradeep@iitm.ac.in. Fax: +91-44 2257-0545.

\section{Notes}

The authors declare no competing financial interest.

\section{ACKNOWLEDGMENTS}

We thank the Department of Science and Technology, Government of India for constantly supporting our research program on nanomaterials. I.C. thanks IITM and S.B. thanks CSIR, Govt. of India for research fellowships. Work by U.L. was supported by the U.S. Air Force Office of Scientific Research and by the Office of Basic Energy Sciences of the U.S. D.O.E. under Contract No. FG05-86ER45234.

\section{REFERENCES}

(1) Jin, R. Quantum Sized, Thiolate-Protected Gold Nanoclusters. Nanoscale 2010, 2, 343-362.

(2) Jadzinsky, P. D.; Calero, G.; Ackerson, C. J.; Bushnell, D. A.; Kornberg, R. D. Structure of a Thiol Monolayer-Protected Gold Nanoparticle at 1.1 Å Resolution. Science 2007, 318, 430-433.

(3) Muhammed, M. A. H.; Verma, P. K.; Pal, S. K.; Kumar, R. C. A.; Paul, S.; Omkumar, R. V.; Pradeep, T. Bright, NIR-Emitting $\mathrm{Au}_{23}$ from $\mathrm{Au}_{25}$ : Characterization and Applications Including Biolabeling. Chem.-Eur. J. 2009, 15, 10110-10120.

(4) Negishi, Y.; Nobusada, K.; Tsukuda, T. Glutathione-Protected Gold Clusters Revisited: Bridging the Gap between Gold(I)Thiolate Complexes and Thiolate-Protected Gold Nanocrystals. J. Am. Chem. Soc. 2005, 127, 5261-5270.

(5) Pei, Y.; Gao, Y.; Zeng, X. C. Structural Prediction of ThiolateProtected $\mathrm{Au}_{38}$ : A Face-Fused Bi-Icosahedral Au Core. J. Am. Chem. Soc. 2008, 130, 7830-7832.

(6) Shibu, E. S.; Pradeep, T. Quantum Clusters in Cavities: Trapped $\mathrm{Au}_{15}$ in Cyclodextrins. Chem. Mater. 2011, 23, 989-999.

(7) Zeng, C.; Qian, H.; Li, T.; Li, G.; Rosi, N. L.; Yoon, B.; Barnett, R. N.; Whetten, R. L.; Landman, U.; Jin, R. Total Structure and Electronic Properties of the Gold Nanocrystal $\mathrm{Au}_{36}(\mathrm{SR})_{24}$. Angew. Chem., Int. Ed. 2012, 51, 13114-13118.

(8) Zhu, M.; Aikens, C. M.; Hollander, F. J.; Schatz, G. C.; Jin, R. Correlating the Crystal Structure of A Thiol-Protected $\mathrm{Au}_{25}$ Cluster and Optical Properties. J. Am. Chem. Soc. 2008, 130, 5883-5885.

(9) Chakraborty, I.; Govindarajan, A.; Erusappan, J.; Ghosh, A.; Pradeep, T.; Yoon, B.; Whetten, R. L.; Landman, U. The Superstable $25 \mathrm{kDa}$ Monolayer Protected Silver Nanoparticle: Measurements and Interpretation as an Icosahedral $\mathrm{Ag}_{152}\left(\mathrm{SCH}_{2} \mathrm{CH}_{2} \mathrm{Ph}\right)_{60}$ Cluster. Nano Lett. 2012, 12, 5861-5866.

(10) Chakraborty, I.; Udayabhaskararao, T.; Pradeep, T. High Temperature Nucleation and Growth of Glutathione Protected $\sim \mathrm{Ag}_{75}$ Clusters. Chem. Commun. 2012, 48, 6788-6790.

(11) Rao, T. U. B.; Nataraju, B.; Pradeep, T. $\mathrm{Ag}_{9}$ Quantum Cluster through a Solid-State Route. J. Am. Chem. Soc. 2010, 132, 1630416307.

(12) Rao, T. U. B.; Pradeep, T. Luminescent $\mathrm{Ag}_{7}$ and $\mathrm{Ag}_{8}$ Clusters by Interfacial Synthesis. Angew. Chem., Int. Ed. 2010, 49, 3925-3929.

(13) Yang, H.; Lei, J.; Wu, B.; Wang, Y.; Zhou, M.; Xia, A.; Zheng, L.; Zheng, N. Crystal Structure of a Luminescent Thiolated Ag Nanocluster with an Octahedral $\mathrm{Ag}_{6}{ }^{4+}$ Core. Chem. Commun. 2012, 49, 300-302.

(14) Chakraborty, I.; Udayabhaskararao, T.; Pradeep, T. Luminescent Sub-nanometer Clusters for Metal Ion Sensing: A New Direction in Nanosensors. J. Hazard. Mater. 2012, 211-212, 396-403.

(15) Lin, C.-A. J.; Yang, T.-Y.; Lee, C.-H.; Huang, S. H.; Sperling, R. A.; Zanella, M.; Li, J. K.; Shen, J.-L.; Wang, H.-H.; Yeh, H.-I.; Parak, W. J.; Chang, W. H. Synthesis, Characterization, and Bioconjugation of Fluorescent Gold Nanoclusters toward Biological Labeling Applications. ACS Nano 2009, 3, 395-401.

(16) Kong, Y.; Chen, J.; Gao, F.; Brydson, R.; Johnson, B.; Heath, G.; Zhang, Y.; Wu, L.; Zhou, D. Near-Infrared Fluorescent RibonucleaseA-Encapsulated Gold Nanoclusters: Preparation, Characterization, Cancer Targeting and Imaging. Nanoscale 2013, 5, 1009-1017.

(17) Nie, X.; Qian, H.; Ge, Q.; Xu, H.; Jin, R. CO Oxidation Catalyzed by Oxide-Supported $\mathrm{Au}_{25}(\mathrm{SR})_{18}$ Nanoclusters and Identification of Perimeter Sites as Active Centers. ACS Nano 2012, 6, $6014-6022$. 
(18) Schmid, G.; Baumle, M.; Geerkens, M.; Heim, I.; Osemann, C.; Sawitowski, T. Current and Future Applications of Nanoclusters. Chem. Soc. Rev. 1999, 28, 179-185.

(19) Anema, J. R.; Li, J.-F.; Yang, Z.-L.; Ren, B.; Tian, Z.-Q. ShellIsolated Nanoparticle-Enhanced Raman Spectroscopy: Expanding the Versatility of Surface-Enhanced Raman Scattering. Annu. Rev. Anal. Chem. 2011, 4, 129-150.

(20) Fan, M.; Andrade, G. F. S.; Brolo, A. G. A Review on the Fabrication of Substrates for Surface Enhanced Raman Spectroscopy and Their Applications in Analytical Chemistry. Anal. Chim. Acta 2011, 693, 7-25.

(21) Kim, K.; Shin, K. S. Surface-Enhanced Raman Scattering: A Powerful Tool for Chemical Identification. Anal. Sci. 2011, 27, 775783.

(22) McNay, G.; Eustace, D.; Smith, W. E.; Faulds, K.; Graham, D. Surface-Enhanced Raman Scattering (SERS) and Surface-Enhanced Resonance Raman Scattering (SERRS): A Review of Applications. Appl. Spectrosc. 2011, 65, 825-837.

(23) Stiles, P. L.; Dieringer, J. A.; Shah, N. C.; Van Duyne, R. P. Surface-Enhanced Raman Spectroscopy. Annu. Rev. Anal. Chem. 2008, $1,601-626$.

(24) Wang, Y.; Yan, B.; Chen, L. SERS Tags: Novel Optical Nanoprobes for Bioanalysis. Chem. Rev. 2013, 113, 1391-1428.

(25) Sonntag, M. D.; Klingsporn, J. M.; Garibay, L. K.; Roberts, J. M.; Dieringer, J. A.; Seideman, T.; Scheidt, K. A.; Jensen, L.; Schatz, G. C.; Van Duyne, R. P. Single-Molecule Tip-Enhanced Raman Spectroscopy. J. Phys. Chem. C 2011, 116, 478-483.

(26) Moore, J. E.; Morton, S. M.; Jensen, L. Importance of Correctly Describing Charge-Transfer Excitations for Understanding the Chemical Effect in SERS. J. Phys. Chem. Lett. 2012, 3, 2470-2475.

(27) He, J.; Lin, X.-M.; Divan, R.; Jaeger, H. M. In-Situ Partial Sintering of Gold-Nanoparticle Sheets for SERS Applications. Small 2011, 7, 3487-3492.

(28) Mullin, J.; Schatz, G. C. Combined Linear Response Quantum Mechanics and Classical Electrodynamics (QM/ED) Method for the Calculation of Surface-Enhanced Raman Spectra. J. Phys. Chem. A 2012, 116, 1931-1938.

(29) Dass, A. Mass Spectrometric Identification of $\mathrm{Au}_{68}(\mathrm{SR})_{34}$ Molecular Gold Nanoclusters with 34-Electron Shell Closing. J. Am. Chem. Soc. 2009, 131, 11666-11667.

(30) Kumar, G. V. P.; Shruthi, S.; Vibha, B.; Reddy, B. A. A.; Kundu, T. K.; Narayana, C. Hot Spots in Ag Core-Au Shell Nanoparticles Potent for Surface-Enhanced Raman Scattering Studies of Biomolecules. J. Phys. Chem. C 2007, 111, 4388-4392.

(31) Shibu, E. S.; Kimura, K.; Pradeep, T. Gold Nanoparticle Superlattices: Novel Surface Enhanced Raman Scattering Active Substrates. Chem. Mater. 2009, 21, 3773-3781.

(32) Stamplecoskie, K. G.; Scaiano, J. C.; Tiwari, V. S.; Anis, H. Optimal Size of Silver Nanoparticles for Surface-Enhanced Raman Spectroscopy. J. Phys. Chem. C 2011, 115, 1403-1409.

(33) Diez, I.; Kanyuk, M. I.; Demchenko, A. P.; Walther, A.; Jiang, H.; Ikkala, O.; Ras, R. H. A. Blue, Green and Red Emissive Silver Nanoclusters Formed in Organic Solvents. Nanoscale 2012, 4, 44344437.

(34) Shibu, E. S.; Radha, B.; Verma, P. K.; Bhyrappa, P.; Kulkarni, G. U.; Pal, S. K.; Pradeep, T. Functionalized $\mathrm{Au}_{22}$ Clusters: Synthesis, Characterization, and Patterning. ACS Appl. Mater. Interfaces 2009, 1, 2199-210.

(35) Dieringer, J. A.; Wustholz, K. L.; Masiello, D. J.; Camden, J. P.; Kleinman, S. L.; Schatz, G. C.; Van Duyne, R. P. Surface-Enhanced Raman Excitation Spectroscopy of a Single Rhodamine 6G Molecule. J. Am. Chem. Soc. 2008, 131, 849-854.

(36) Majoube, M. Vibrational Spectra of Adenine and DeuteriumSubstituted Analogues. J. Raman Spectrosc. 1985, 16, 98-110.

(37) Johnson, B. B.; Peticolas, W. L. The Resonant Raman Effect. Annu. Rev. Phys. Chem. 1976, 27, 465-521.

(38) Kleinman, S. L.; Ringe, E.; Valley, N.; Wustholz, K. L.; Phillips, E.; Scheidt, K. A.; Schatz, G. C.; Van Duyne, R. P. Single-Molecule Surface-Enhanced Raman Spectroscopy of Crystal Violet Isotopo- logues: Theory and Experiment. J. Am. Chem. Soc. 2011, 133, 41154122.

(39) Kneipp, K.; Kneipp, H.; Itzkan, I.; Dasari, R. R.; Feld, M. S. Ultrasensitive Chemical Analysis by Raman Spectroscopy. Chem. Rev. 1999, 99, 2957-2976.

(40) Kim, H.; Kosuda, K. M.; Van Duyne, R. P.; Stair, P. C. Resonance Raman and Surface- And Tip-Enhanced Raman Spectroscopy Methods to Study Solid Catalysts and Heterogeneous Catalytic Reactions. Chem. Soc. Rev. 2010, 39, 4820-4844.

(41) Huang, R.; Zhao, L.-B.; Wu, D.-Y.; Tian, Z.-Q. Tautomerization, Solvent Effect and Binding Interaction on Vibrational Spectra of Adenine- $\mathrm{Ag}^{+}$Complexes on Silver Surfaces: A DFT Study. J. Phys. Chem. C 2011, 115, 13739-13750.

(42) Muniz-Miranda, M.; Gellini, C.; Pagliai, M.; Innocenti, M.; Salvi, P. R.; Schettino, V. SERS and Computational Studies on MicroRNA Chains Adsorbed on Silver Surfaces. J. Phys. Chem. C 2010, 114, 13730-13735.

(43) Papadopoulou, E.; Bell, S. E. J. Structure of Adenine on Metal Nanoparticles: $\mathrm{pH}$ Equilibria and Formation of $\mathrm{Ag}^{+}$Complexes Detected by Surface-Enhanced Raman Spectroscopy. J. Phys. Chem. C 2010, 114, 22644-22651.

(44) Papadopoulou, E.; Bell, S. E. J. Surface Enhanced Raman Evidence for $\mathrm{Ag}^{+}$Complexes of Adenine, Deoxyadenosine and 5'dAMP Formed in Silver Colloids. The Analyst 2010, 135, 3034-3037. 